\title{
Thyroid Stimulating Hormone, Cognitive Impairment and Depression in an Older Korean Population
}

\author{
Jae-Min Kim¹, Robert Stewart², Seon-Young Kim¹, Kyung-Yeol Bae', \\ Su-Jin Yang', Sung-Wan Kim¹, II-Seon Shin ${ }^{1 凶}$ and Jin-Sang Yoon ${ }^{1}$ \\ 1Department of Psychiatry and Center for Aging \& Geriatrics (BK21 Program), Chonnam National University Medical School, Gwangju, Korea \\ 2Section of Epidemiology, Institute of Psychiatry, London, United Kingdom
}

Objective Associations of thyroid dysfunction with cognitive impairment and depression in late-life have been described but remain controversial. This study aimed to investigate the associations of serum thyroid stimulating hormone (TSH) levels with cognitive impairment and depression after controlling for potential confounding factors.

Methods The sample consisted of 495 community residents aged 65 or over in whom serum TSH had been assayed. Cognitive impairment was defined using the Community Screening Interview for Dementia, and depression was diagnosed using the Geriatric Mental State schedule. Age, gender, education, smoking history, physical activity, blood pressure, diabetes, and serum total cholesterol and albumin were included as covariates.

Results There was a significant association between lower (hyperthyroid) serum TSH levels $(<0.5 \mathrm{mIU} / \mathrm{L})$ and cognitive impairment after adjustment [odds ratio 7.12 (95\% confidence interval 1.35-37.5)]. However, no association was found between TSH levels and depression.

Conclusion Based on TSH levels, hyperthyroidism but not hypothyroidism was associated with cognitive impairment in this sample, and we found no evidence for an association of either with depression.

Psychiatry Investig 2010;7:264-269

Key Words Aging, Cognition, Depression, Thyroid stimulating hormone, Korea.

\section{INTRODUCTION}

Thyroid dysfunction is common, particularly in older people. ${ }^{1}$ Considerable efforts have been made to elucidate associations of thyroid dysfunction with both somatic and mental disorders, but results have been controversial. ${ }^{2}$ In the field of geriatric psychiatry, associations with cognitive function and depression have received particular attention. Thyroid stimulating hormone (TSH) is a sensitive marker for abnormal thyroid function, and therefore most studies have included this assay.,

With respect to cognitive function in older people, some studies have found low serum TSH levels to be associated with

Received: April 22, 2010 Revised: July 23, 2010

Accepted: August 9, 2010 Available online: November 30, 2010

$\triangle$ Correspondence: Il-Seon Shin, $\mathrm{MD}, \mathrm{PhD}$

Department of Psychiatry and Center for Aging \& Geriatrics (BK21 Program), Chonnam National University Medical School, 5 Hak-dong, Dong-gu, Gwangju 501-757, Korea

Tel: +82-61-379-7762, Fax: +82-62-225-2351, E-mail: isshin@chonnam.ac.kr (c) This is an Open Access article distributed under the terms of the Creative Commons Attribution Non-Commercial License (http://creativecommons.org/licenses/by$\mathrm{nc} / 3.0$ ) which permits unrestricted non-commercial use, distribution, and reproduction in any medium, provided the original work is properly cited. cognitive impairment or dementia, ${ }^{5,6}$ others have found no associations, ${ }^{7,8}$ and others have found high TSH levels to be associated with worse working memory., ${ }^{910}$ The Framingham Study found that both low and high TSH levels were associated with an increased risk of incident dementia. ${ }^{11}$

Regarding late-life depression, studies have found associations between high serum TSH levels and depression ${ }^{12}$ as well as an increased risk of hospitalization with affective disorder. ${ }^{13}$ However, other studies have found no associations between thyroid dysfunction and depression. ${ }^{7,8,14,15}$

Overall, these research questions remain controversial and require further investigation. Using data from a sample of community dwelling Korean elders, we investigated associations of serum TSH levels with cognitive impairment and depression after controlling for potential confounders.

\section{METHODS}

\section{Study area and population}

In collaboration with the 10/66 Dementia in Developing Countries Research Program, ${ }^{16}$ several consecutive communi- 
ty surveys of psychiatric morbidity in late-life have been carried out in Gwangju, South Korea from 2001 to 2006. Similar protocols were administered for the studies, and the study results from data obtained in 2001 and 2003 have been published previously. ${ }^{17,18}$ Serum TSH levels were assayed in a further crosssectional survey conducted in 2004. A geographic catchment area design was used to obtain the sample. This area, Hakwoon, is located in the inner, very urbanized compartment of Gwangju city. It covers a land area of $11 \mathrm{~km}^{2}$ and has an estimated population of 14,518 of whom 1,499 (10.4\%) were known to be aged 65 or over from national registration lists. Potential participants for the study were recruited from this latter group.

\section{Interviews and procedures}

Before the survey, a letter explaining the study was sent to all potential participants. Contact was made by home visits, repeated at least two times if no reply was obtained. Single stage assessments for interview, measurements and blood sampling were carried out by three research nurses who were trained for this particular study and supervised by the project psychiatrist throughout the survey period. All participants gave informed consent, and the study was approved by the Chonnam National University Hospital Institutional Review Board.

\section{Serum TSH and classification of thyroid function}

Blood samples were collected in a fasting state, carried out in the mornings where possible. Serum TSH levels were meas ured by using a chemiluminescent immunoassay (Cobas: Roche Diagnotics, West Sussex, UK) in the Clinical Laboratory of Chonnam National University Hospital. The laboratory reference range was $0.50-4.50 \mathrm{mIU} / \mathrm{L}$. For the analyses presented here, we classified thyroid function according to serum levels of TSH: 1) hyperthyroid state ( $<0.50 \mathrm{mIU} / \mathrm{L}) ; 2)$ euthyroid state (0.50-4.50 mIU/L); and 3) hypothyroid state $(>4.50$ $\mathrm{mIU} / \mathrm{L})$. The euthyroid state was further divided into tertiles for additional analysis. Use of levothyroxine was ascertained from inventories taken of medication use in survey participants.

\section{Cognitive impairment}

Cognitive function was evaluated using the Community Screening Interview for Dementia (CSID), which is a 32-item cognitive test administered to the participants (20 $\mathrm{min}$ ) and 26-item informant interview about the participants daily functioning and general health lasting around 15 minutes. ${ }^{19}$ Three summary scores can be generated from CSID: 1) the cognitive score (COGSCORE), an item-weighted total score from the participants cognitive test; 2) the informant score (RELSCORE), an unweighted total score from the informant interview; and 3) the discriminant function score (DFSCORE), a weighted score combining COGSCORE and RELSCORE. DFSCORE has a validated cutoff, and has achieved $87 \%$ sensitivity and $83 \%$ specificity for a clinical diagnosis of DSM-III-R dementia. ${ }^{19}$ The CSID was translated into Korean (CSID-K), and its reliability and validity in this language have been previously evaluated and described. ${ }^{20}$ The standard cut-off for the DFSCORE was administered to define cognitive impairment in the study.

\section{Depression}

Depression was assessed using the community version of the Geriatric Mental State diagnostic schedule (GMS B3). ${ }^{21}$ This is a fully structured diagnostic instrument in wide international use with an accompanying computerised algorithm. The GMS B3 was translated into Korean according to a formal standardization process. ${ }^{22}$ Diagnosis of depression in the last month was generated using the Automated Geriatric Examination for Computer Assisted Taxonomy (AGECAT) algorithm. Participants rated as AGECAT 3, 4, and 5 are considered to be likely cases, those rated as 1 and 2 are considered to be sub-cases, and those rated as 0 to have no relevant symptomatology. As in other studies, a 'stage one' (non-hierarchical) confidence level of 3 or above in the AGECAT algorithm was used in this study to define depression of clinical significance.

\section{Other covariates}

Demographic data on age, gender, and education were recorded. Smoking history was ascertained. Daily physical activity, taking into account both work and leisure activity, was ascertained and sedentary lifestyle was defined as a binary variable. Resting blood pressure (BP) was taken with an automatic sphygmomanometer on the left arm in the sitting position. Self-reported diagnosis of and treatment history for diabetes were recorded. Blood assays for total cholesterol and albumin were conducted, as these had been found to be potential confounding factors in other similar studies. ${ }^{7,8}$

\section{Statistical analyses}

Statistical analyses were carried out using SPSS 12.0 software. Data on demographic and clinical characteristics by the categories of thyroid function were compared using analysis of variance or $\chi^{2}$ (Fisher's exact) tests as appropriate. Unadjusted associations of serum TSH levels (from lower to higher) with CSID caseness and GMS depression were estimated by $\chi^{2}$ tests (linear terms). For regression analyses, the middle 2 nd tertile of the euthyroid state was treated as reference category. Four adjusted models were used for each outcome: model 1 included age, gender and education; model 2 included model 1 plus smoking and physical activity; model 3 included model 2 plus systolic BP, diabetes mellitus, total cholesterol, and albumin; and model 4 includes model 3 plus levothyroxine treatment and depression. 


\section{RESULTS}

\section{Recruitment}

Of 1,499 inhabitants aged 65 or over identified from registration lists, 766 (51\%) completed the interview with research nurses. Of the remainder, contact could not be established with 447 (30\%), 225 (15\%) refused to participate by elders or their family members, 54 (3\%) had no fixed abode, and 7 (1\%) had died before the visit. No significant differences were observed in age (mean ages, 72.6 and 72.9 respectively) and gender ratio (60\% and 56\% female, respectively) between participants and non-participants (all p-values $>0.1$ ).

Of 766 participants to the interview, 495 (65\%) agreed to a blood test and comprised the study sample. No significant differences were observed between the participants and non-participants at this stage in terms of age (mean ages, 72.4 and 72.9 respectively), gender (61\% and 59\% female), education (mean years, 8.0 and 8.1), CSID caseness (8.7\% and 9.6\%), and GMS depression (15.8\% and 15.6\%) (all p-values $>0.1$ ).

\section{Characteristics of the participants by thyroid function}

Thyroid function categories within the sample are described in the first through third rows of Table 1 . According to the serum TSH levels, $3 \%$ of the participants were in a hyperthyroid state, $7 \%$ were in a hypothyroid state, and $90 \%$ were euthyroid. The mean (SD) TSH level was 2.1 (1.8) mIU/L. Levothyroxine was taken by 36 (7\%) participants. Other demographic and clinical characteristics are compared by thyroid states in the fourth through last rows of Table 1 . The proportion of current smokers was significantly higher in the hyperthyroid group. However, no other differences were found between categories.

\section{Unadjusted associations with cognitive impairment and depression}

Unadjusted associations of thyroid function with cognitive impairment and depression are illustrated in Figure 1. The prevalence of cognitive impairment was highest in the hyperthyroid group, and gradually decreased across euthyroid to hypothyroid ranges. However, the prevalence of depression did not markedly change across the range of thyroid function.

\section{Adjusted associations with cognitive impairment and depression}

Regression analyses for cognitive impairment as an outcome are summarised in Table 2. Compared to the reference category (2nd tertile of euthyroid state), cognitive impairment was significantly raised in the hyperthyroid group, but did not significantly differ across other categories, although odds ratios remained in the directions originally observed and strengths of association were increased in the adjusted models. In a further regression analysis on entering the thyroid function categories as an ordinal variable on one degree of freedom, the odds ratio (95\% confidence interval) for cognitive impairment was 1.61 (1.12-2.30) per group increment in the full adjusted model. No significant associations were found in regression analyses between thyroid function categories and depression (data not shown).

\section{DISCUSSION}

In a community dwelling Korean elderly population, we found a significant association between lower serum TSH levels $(<0.5 \mathrm{mIU} / \mathrm{L})$ indicative of hyperthyroidism and cognitive

Table 1. Characteristics of the participants by thyroid status

\begin{tabular}{|c|c|c|c|c|c|c|c|}
\hline & \multirow{2}{*}{$\begin{array}{c}\text { Total } \\
\text { participants }\end{array}$} & \multirow{2}{*}{$\begin{array}{c}\text { Hyperthyroid } \\
\text { state }\end{array}$} & \multicolumn{3}{|c|}{ Euthyroid state } & \multirow{2}{*}{$\begin{array}{l}\text { Hypothyroid } \\
\text { state }\end{array}$} & \multirow{2}{*}{ p-value* } \\
\hline & & & Tertile 1 & Tertile 2 & Tertile 3 & & \\
\hline articipants, N (\%) & $495(100)$ & $14(3)$ & $147(30)$ & $149(30)$ & $148(30)$ & $37(7)$ & \\
\hline TSH level, mIU/L range & $0.02-17.21$ & $0.02-0.49$ & $0.50-1.28$ & $1.29-1.96$ & $1.97-4.50$ & $4.51-17.21$ & \\
\hline Levothyroxine treatment, $\mathrm{N}(\%)$ & $36(7)$ & $1(7)$ & $9(6)$ & $12(8)$ & $11(7)$ & $3(8)$ & 0.655 \\
\hline Age, mean $(\mathrm{SD})$ years & $72.4(5.6)$ & $73.8(5.4)$ & $72.1(5.6)$ & $72.6(5.9)$ & $72.2(5.4)$ & $73.2(5.8)$ & 0.633 \\
\hline Gender, N (\%) female & $301(61)$ & $7(50)$ & $88(60)$ & $89(60)$ & $89(60)$ & $28(76)$ & 0.185 \\
\hline Education, mean (SD) years & $8.0(5.6)$ & $8.0(6.9)$ & $8.4(5.7)$ & $7.7(5.5)$ & $8.1(5.8)$ & $7.0(5.1)$ & 0.623 \\
\hline Current smoker, N (\%) & $145(29)$ & $8(57)$ & $45(31)$ & $49(33)$ & $39(26)$ & $4(11)$ & 0.006 \\
\hline Physical inactivity, N (\%) & $153(31)$ & $4(29)$ & $42(29)$ & $48(32)$ & $45(30)$ & $14(38)$ & 0.410 \\
\hline Systolic BP, mean (SD) mmHg & $138.2(20.4)$ & $142.6(19.3)$ & $135.3(18.6)$ & $141.3(21.7)$ & $138.0(19.9)$ & $136.2(23.1)$ & 0.121 \\
\hline Diastolic BP, mean (SD) mmHg & $80.6(11.6)$ & $82.4(8.7)$ & $79.5(11.5)$ & $81.1(12.1)$ & $81.1(11.2)$ & $79.8(12.8)$ & 0.663 \\
\hline Diabetes mellitus, N (\%) & $84(17)$ & $4(29)$ & $18(12)$ & $26(17)$ & $31(21)$ & $5(14)$ & 0.401 \\
\hline Total cholesterol, mean (SD) mg/dL & $178.8(36.4)$ & $178.7(35.3)$ & $180.7(37.2)$ & $177.1(36.5)$ & $176.3(33.8)$ & $187.6(43.2)$ & 0.465 \\
\hline Albumin, mean (SD) mg/dL & $4.3(0.4)$ & $4.3(0.3)$ & $4.2(0.4)$ & $4.3(0.4)$ & $4.3(0.4)$ & $4.4(0.3)$ & 0.715 \\
\hline
\end{tabular}

*analysis of variance or $\chi^{2}$ (Fisher's exact) tests as appropriate. TSH: thyroid stimulating hormone, BP: blood pressure 
impairment, while no associations were found between TSH levels and depression.

Serum TSH assay is very responsive to minute changes in circulating thyroid hormone levels and so it is a sensitive and specific test. ${ }^{4}$ In the present study, a hyperthyroid state was present in $3 \%$ and hypothyroid state in $7 \%$ of the participants. These were similar to other previous studies with older community samples, where the below normal (hyperthyroid) TSH levels have been described in 2.6-5.5\% and above normal (hypothyroid) TSH in 3.3-12.0\%.$^{6.8}$ Also $7 \%$ of the present sample were on levothyroxine, which were similar to reports from other studies with older samples of $4-11 \% .1,15$

There was a significant association between below normal (hyperthyroid) serum TSH levels and cognitive impairment. Furthermore, a significant linear association between lower TSH levels and cognitive impairment was observed. These results are consistent with the previous studies reporting associations between low TSH levels and cognitive impairment ${ }^{5}$ or incident dementia. ${ }^{6}$ Several plausible mechanisms for the associations have been suggested. Thyroid hormones induce deposition of $\beta$-amyloid changes, ${ }^{23}$ and higher thyroid hormone levels have been found to be associated with a higher count of

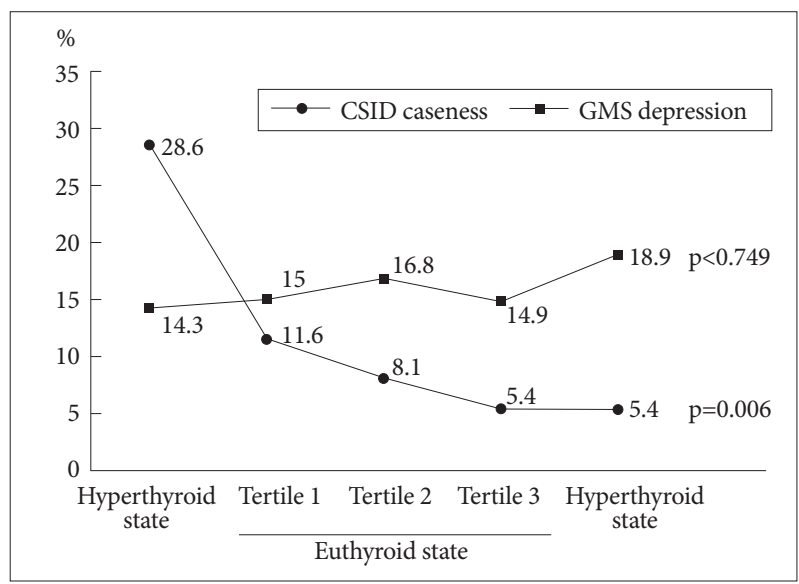

Figure 1. Unadjusted associations between thyroid function, cognitive impairment and depression. CSID: Community Screening Interview for Dementia, GMS: Geriatric Mental state Schedule. neocortical neuritic plaque ${ }^{24}$ and smaller hippocampal volume. ${ }^{25}$ However, some large epidemiological studies of older populations have failed to find this association..$^{7.8}$ Discrepant findings might be due to the fact that the proportions of participants with below or above normal TSH levels are relatively small in each study, and so results may vary related to low statistical power. In addition, TSH values may be altered by as much as $30 \%$ depending on time of day of phlebotomy, and the fasting or non-fasting status of participants. ${ }^{26}$ Previous studies finding no associations have tended to be those which have analyzed random TSH measures. ${ }^{7.8}$ Consistency of blood sampling times is challenging for epidemiological research, but blood samples in this study were collected in a fasting state and phlebotomy was carried out in the mornings where possible.

There have been some studies finding associations between thyroid dysfunction and affective disorders including depression, ${ }^{27}$ while others have found no associations. ${ }^{7,8,14,15}$ Inconsistent results might be due to differences in study setting (clinical or community), depression identification (diagnostic instrument or assessment scale), consideration of confounding factors, and different TSH assays. Previous studies reporting significant associations have tended to use clinical samples ${ }^{12,28}$ and may not be generalisable to source communities. Epidemiological research on the other hand has tended to use brief screening instruments rather than diagnostic interviews. In the present study, the sample was drawn from community dwelling elders, and depression was also diagnosed using a fully structured instrument which itself has been widely used in international epidemiological research. ${ }^{29}$

We incidentally found a significant association between hyperthyroid state and current smoking in the present study. Our study was cross-sectional in design, and therefore could not reveal the causal relationship between thyroid problem and smoking status. ${ }^{30}$ However, this finding was consistent with the previous well known study reporting that smoking was a risk factor for hyperthyroidism. ${ }^{31}$

Strengths of the study were that cases of cognitive impairment and depression were identified by well recognized and for-

Table 2. Multi-variate associations with cognitive impairment. Data are odds ratios (95\% confidence intervals)

\begin{tabular}{|c|c|c|c|c|c|c|}
\hline & & \multirow{2}{*}{ Unadjusted } & \multicolumn{4}{|c|}{ Adjusted } \\
\hline & & & Model 1 & Model 2 & Model 3 & Model 4 \\
\hline Hyperthyroid state & & $4.57(1.24-16.8)$ & $5.42(1.20-24.4)$ & $6.30(1.20-33.0)$ & $7.05(1.36-36.6)$ & $7.12(1.35-37.5)$ \\
\hline \multirow[t]{3}{*}{ Euthyroid state } & Tertile 1 & $1.49(0.69-3.25)$ & $1.73(0.75-3.98)$ & $1.89(0.80-4.50)$ & $1.99(0.82-4.84)$ & $1.97(0.81-4.81)$ \\
\hline & Tertile 2 & 1.00 (ref) & 1.00 (ref) & 1.00 (ref) & $1.00(\mathrm{ref})$ & $1.00(\mathrm{ref})$ \\
\hline & Tertile 3 & $0.65(0.26-1.65)$ & $0.67(0.25-1.76)$ & $0.69(0.25-1.90)$ & $0.64(0.23-1.79)$ & $0.63(0.22-1.76)$ \\
\hline Hypothyroid state & & $0.65(0.14-3.05)$ & $0.51(0.10-2.55)$ & $0.49(0.09-2.57)$ & $0.42(0.08-2.30)$ & $0.45(0.08-2.49)$ \\
\hline
\end{tabular}

Model 1 includes age, gender and education, Model 2 includes Model 1+smoking and physical activity, Model 3 includes Model 2+systolic blood pressure, diabetes mellitus, total cholesterol and albumin, Model 4 includes Model $3+$ levothyroxine treatment and depression 
mally validated instruments, that the study sample was drawn from a community population, and that important factors potentially affecting serum TSH levels were considered in the analyses as potential confounding factors. An important limitation of the study was that measures of thyroid function other than TSH were not available in the study. In particular in the relationships with depression and dementia, it is important to distinguish subclinical and clinical thyroid dysfunction, also to see whether the effects are dose-dependent, while it was impossible in this study due to its limited design. The participation rate of the study was also low. The main reason for nonparticipation was that contact could not be established and the reason for this is most likely to be incorrect registrations on the national database-in particular, elders registering in urban areas where their siblings reside, but actually living themselves in rural areas. In previous surveys in this region which have included both urban and rural samples, participation rates in urban residents have been found to be substantially lower than those in rural areas. ${ }^{32}$

Thyroid dysfunction, cognitive impairment, and depression are common problems in old individuals. The possible associations of thyroid dysfunction with cognitive impairment and depression are very important, because management of thyroid dysfunction is likely to have beneficial effects on both somatic and mental health. Furthermore, treatment of thyroid dysfunction is relatively straightforward. However, further studies are still needed to investigate the association more mechanistically.

\section{Acknowledgments}

This research was supported by a grant of the Korea Health 21 R\&D Project, Ministry of Health and Welfare, Republic of Korea (A050047).

\section{REFERENCES}

1. Canaris GJ, Manowitz NR, Mayor G, Ridgway EC. The Colorado thyroid disease prevalence study. Arch Intern Med 2000;160:526-534.

2. Surks MI, Ortiz E, Daniels GH, Sawin CT, Col NF, Cobin RH, et al. Subclinical thyroid disease: scientific review and guidelines for diagnosis and management. JAMA 2004;291:228-238.

3. Biondi B, Cooper DS. The clinical significance of subclinical thyroid dysfunction. Endocr Rev 2008;29:76-131.

4. Rodondi N, Newman AB, Vittinghoff E, de Rekeneire N, Satterfield S, Harris TB, et al. Subclinical hypothyroidism and the risk of heart failure, other cardiovascular events, and death. Arch Intern Med 2005;165: 2460-2466.

5. Ceresini G, Lauretani F, Maggio M, Ceda GP, Morganti S, Usberti E, et al. Thyroid function abnormalities and cognitive impairment in elderly people: results of the Invecchiare in Chianti study. J Am Geriatr Soc 2009; 57:89-93.

6. Kalmijn S, Mehta KM, Pols HA, Hofman A, Drexhage HA, Breteler MM. Subclinical hyperthyroidism and the risk of dementia. The Rotterdam study. Clin Endocrinol (Oxf) 2000;53:733-737.

7. Gussekloo J, van Exel E, de Craen AJ, Meinders AE, Frölich M, Westendorp RG. Thyroid status, disability and cognitive function, and survival in old age. JAMA 2004;292:2591-2599.
8. Roberts LM, Pattison H, Roalfe A, Franklyn J, Wilson S, Hobbs FD, et al. Is subclinical thyroid dysfunction in the elderly associated with depression or cognitive dysfunction? Ann Intern Med 2006;145:573-581.

9. Samuels MH, Schuff KG, Carlson NE, Carello P, Janowsky JS. Health status, mood, and cognition in experimentally induced subclinical hypothyroidism. J Clin Endocrinol Metab 2007;92:2545-2551.

10. Zhu DF, Wang ZX, Zhang DR, Pan ZL, He S, Hu XP, et al. fMRI revealed neural substrate for reversible working memory dysfunction in subclinical hypothyroidism. Brain 2006;129:2923-2930.

11. Tan ZS, Beiser A, Vasan RS, Au R, Auerbach S, Kiel DP, et al. Thyroid function and the risk of Alzheimer disease: the Framingham Study. Arch Intern Med 2008;168:1514-1520.

12. Chueire VB, Romaldini JH, Ward LS. Subclinical hypothyroidism increases the risk for depression in the elderly. Arch Gerontol Geriatr 2007; 44:21-28.

13. Thomsen AF, Kvist TK, Andersen PK, Kessing LV. Increased risk of developing affective disorder in patients with hypothyroidism: a registerbased study. Thyroid 2005;15:700-707.

14. Engum A, Bjøro T, Mykletun A, Dahl AA. An association between depression, anxiety and thyroid function--a clinical fact or an artefact? Acta Psychiatr Scand 2002;106:27-34.

15. Fraser SA, Kroenke K, Callahan CM, Hui SL, Williams JW Jr, Unützer J. Low yield of thyroid-stimulating hormone testing in elderly patients with depression. Gen Hosp Psychiatry 2004;26:302-309.

16. Prince M, Acosta D, Chiu H, Scazufca M, Varghese M; 10/66 Dementia Research Group. Dementia diagnosis in developing countries: a crosscultural validation study. Lancet 2003;361:909-917.

17. Kim JM, Stewart R, Shin IS, Yoon JS. Vascular disease/risk and late-life Depression in a Korean community population. Br J Psychiatry 2004;185: 102-107.

18. Kim JM, Stewart R, Kim SW, Yang SJ, Shin IS, Kim YH, et al. Interactions between life stressors and susceptibility genes (5-HTTLPR and BDNF) on depression in Korean elders. Biol Psychiatry 2007;62:423-428.

19. Hall KS, Hendrie HC, Brittain HM, Norton JA. The development of a dementia screening interview in two distinct languages. Int J Meth Psychiatr Res 1993;3:1-28.

20. Kim JM, Kim SW, Shin IS, Zheng TJ, Yoon JS. Development of Korean version of community screening interview for dementia(CSID-K). J Korean Neuropsychiatr Assoc 2004;43:445-451.

21. Copeland JR, Dewey ME, Griffiths-Jones HM. A computerized psychiatric diagnostic system and case nomenclature for elderly subjects: GMS and AGECAT. Psychol Med 1986;16:89-99.

22. Kim JM, Stewart R, Prince M, Shin IS, Yoon JS. Diagnosing dementia in a developing nation: an evaluation of the GMS-AGECAT algorithm in an older Korean population. Int J Geriatr Psychiatry 2003;18:331-336.

23. Latasa MJ, Belandia B, Pascual A. Thyroid hormones regulate beta-amyloid gene splicing and protein secretion in neuroblastoma cells. Endocrinology 1998;139:2692-2698.

24. de Jong FJ, Masaki K, Chen H, Remaley AT, Breteler MM, Petrovitch $\mathrm{H}$, et al. Thyroid function, the risk of dementia and neuropathologic changes: the Honolulu-Asia aging study. Neurobiol Aging 2009;30: 600-606.

25. de Jong FJ, den Heijer T, Visser TJ, de Rijke YB, Drexhage HA, Hofman A, et al. Thyroid hormones, dementia, and atrophy of the medial temporal lobe. J Clin Endocrinol Metab 2006;91:2569-2573.

26. Scobbo RR, VonDohlen TW, Hassan M, Islam S. Serum TSH variability in normal individuals: the influence of time of sample collection. W V Med J 2004;100:138-142.

27. Musselman DL, Nemeroff CB. Depression and endocrine disorders: focus on the thyroid and adrenal system. Br J Psychiatry Suppl 1996;30: 123-128.

28. Haggerty JJ Jr, Stern RA, Mason GA, Beckwith J, Morey CE, Prange AJ Jr. Subclinical hypothyroidism: a modifiable risk factor for depression? Am J Psychiatry 1993;150:508-510.

29. Copeland JRM, Dewey ME, Saunders P. The epidemiology of demen- 
tia: GMS-AGECAT studies of prevalence and incidence, including studies in progress. Eur Arch Psychiatry Clin Neurosci 1991;240:212-217.

30. Utiger RD. Cigarette smoking and the thyroid. N Engl J Med 1995;333: 1001-1002.

31. Holm IA, Manson JE, Michels KB, Alexander EK, Willett WC, Utiger RD.
Smoking and other lifestyle factors and the risk of Graves' hyperthyroidism. Arch Intern Med 2005;165:1606-1611.

32. Kim JM, Stewart R, Shin IS, Yoon JS, Lee HY. Lifetime urban/rural residence, social support and late-life depression in Korea. Int J Geriatr Psychiatry 2004;19:843-851. 